\title{
NUMERICAL STUDY ON THE EFFECT OF AN OFF-SURFACE MICRO-ROD VORTEX GENERATOR PLACED UPSTREAM NACA0012 AEROFOIL
}

\author{
Abderrahim LARABI $^{1}$, Michael PEREIRA ${ }^{2}$, Florent RAVELET $^{2}$, Tarik AZZAM ${ }^{1}$, Hamid OUALLI ${ }^{1}$, Laiche MENFOUKH ${ }^{1}$, and \\ Farid BAKIR $^{2 *}$
}

${ }^{1}$ Fluid Mechanics Laboratory, Ecole Militaire Polytechnique, BP 17 Bordj El Bahri, Algiers, 16111, Algeria

${ }^{2}$ Arts et Metiers Institute of Technology, CNAM, LIFSE, HESAM University, F-75013 Paris, France

\begin{abstract}
In this paper, 3D numerical simulations have been carried out to enhance the understanding of a flow over a passive control device composed of micro cylinder with, $\mathrm{d} / \mathrm{c}=1.34 \%$ placed in the vicinity of NACA0012 aerofoil wing, by means of $\gamma$-Re $\theta_{t}$ transition sensitive turbulence model meant to predict the separation induced by transition achieved for aerofoils operating at moderate Reynolds number $(\operatorname{Re}=$ $\left.4.45 \times 10^{5}\right)$. Results show that the separation of the boundary layer has been eliminated by the passive static vortex generator at stall regime due to the injection of free-stream momentum to the boundary layer. The early transition to turbulent state overcomes the local flow deceleration of an adverse pressure gradient and remains sticked to the wall the boundary layer. Furthermore, the wing aerodynamic performance are improved as drag is reduced and lift is enhanced which is straight forward linked to the lift to drag ratio gain that varies from $22.68 \%$ to $134.17 \%$ at post stall angles of attack.
\end{abstract}

\section{Nomenclature}

$\begin{array}{ll}A O A & \text { Angle of attack } \\ c & \text { Chord length } \\ c D & \text { Drag coefficient } \\ c_{L} & \text { Lift coefficient } \\ C p & \text { Pressure coefficient } \\ d & \text { Rod diameter } \\ k & \text { Turbulent kinetic energy } \\ L & \text { Distance between the Rod and aerofoil } \\ \mathrm{LE} & \\ \mathrm{LE} & \text { Leading Edge } \\ L S B & \text { Laminar Separation Bubble } \\ \operatorname{Re} & \text { Reynolds number } \\ \operatorname{Re} \theta, t & \text { Re number where transition occurs } \\ S S T & \text { Shear Stress Transport } \\ t & \text { Time } \\ \mathbf{U} & \text { Velocity field } \\ V_{\infty} & \text { Freestream velocity } \\ y^{+} & \text {Non-dimentional wall distance } \\ \alpha & \text { Angle of attack } \\ \gamma & \text { Intermittency } \\ \varepsilon & \text { Turbulent dissipation rate } \\ \omega & \text { Specific dissipation rate }\end{array}$

\section{Introduction}

In recent decades, controlling flows has sparked lots of attention in fluid mechanics given its positive impact on industrial applications. Modifying flow field behavior to achieve extreme technologically important benefits such as improved aerodynamic performance, increased heat exchange, intensified turbulent mixing, and reduced vibrations of aeronautical structures. Airfoils are one of the most fundamental components of aircraft and UAVs, and its aerodynamic performance has a direct impact on their efficiency. In particular, they are often subjected to substantial unfavourable adverse pressure gradients, which lead to inevitable flow separation mainly at a high angle of attack. This separation grows bigger as incidence increased to eventually cover the whole suction surface, causing the aerofoil to stall. When a stall occurs, it is characterized by drag increase, lift drop, as well as aerodynamic noise production, that are typically undesirable. Hence, flow control is the proper way for improving the design and achieving the said objectives.

Based on the source and amount of energy involved, flow control can be classified as active, passive or even reactive techniques [1]. Fundamentally, passive flow control techniques are focused to accelerate boundary layer transition from laminar to turbulent state, mainly by modifying geometrical shape of bodies. Therefore, improving wing performance doesn't require to inject an external energy to assist the flow. Generally, these techniques are inspired from nature so that several impressive control mechanisms can be observed as vortex generators such as, fish skin and flippers, bird wings, insect bodies, groovs, roughness elements slots and more. Which are steady integrated to the body (on-surface) itself to alter natural flow state to the desirable condition.

Another passive flow control approach is adopted by introducing off-surface control elements placed beyond the boundary layer based on bodies wake interaction by a mutual interference as the vortex shedding from the element pumps the outer flow momentum to the body boundary layer that efficiently delays its separation. It was found that these kind of vortex generator devices might perform better than integrated onces provided their optimum design settings was carefully adopted. The early application of such control concept was first applied to bulff body throughout experimental and numerical works conducted by Strykowski and Sreenivasan [2] aiming to

\footnotetext{
* Corresponding author: farid.bakir@ensam.eu
} 
suppress its vortex shedding using smaller cylinder in the near wake of the main cylinder. The same idea was encouraged by an experimental study carried out by Sakamoto et al. [3], it was concluded that the influence of tiny cylinder on square prism was significant resulting in mean drag reduction of about $30 \%$. Sakamoto and Haniu [4] have examined the impact of a small rod placed upstream a bigger cylinder at flow conditions of $6.5 \times 10^{4}$. The experiments showed promessing results as maximum drag drop was $50 \%$ as well as damping the fluctuations on both lift and drag forces. A tiny cylinder vortex generator was used by Igarashi [5] to control the flow over a square prism at Reynolds number of $3.2 \times 10^{4}$. It was discovered that when an optimal rod settings are adopted the prism's drag was reduced by roughly $50 \%$. Tsutsui and Igarashi [6] have showed experimentally that when a steady vortex generator rod is set at an optimum conditions of $\mathrm{d} / \mathrm{D}=0.25$ and $\mathrm{L} / \mathrm{D}=1.75-2.0$, it had substantial effect on reducing drag up to $63 \%$ by completely removing the shedding vortices from the cylinder. PIV analysis performed by Michelis and Kotsonis [7] to assess the impact of off-surface rod used to control the flow around a bluff body at Reynolds number of $2.6 \times 10^{4}$, on the mean flow topology. It has been proven that the separation bubble on the cylinder was removed and the vortex shedding pattern alter its state from phase-locked to regular one. Eventhough, the use of a tiny off-surface vortex generator to control the flow over bluff bodies seemed very effective, it cannot be straightforward conclusive for slender bodies like airfoils, where, considerable increase in overall drag of the whole system might be unavoidable at pre-stall regime. To control the separated flow around NACA2412 airfoil, Hayder Kraidi and Rashid Nasrawi [8] have set two neighboring circular cylinders with an equal diameter of $10 \mathrm{~mm}$ upstream of the body. The experimental and numerical results showed that the shedded vortices interact with the boundary layer on the suction side which tends to prevent flow separation, hence pushing the separation point to move downstream. An offsurface micro-cylinder was used by Chen et al. [9] to control the flow over VAWT blade aiming to enhance its power generation. In their numerical investigations, the aerodynamic performances for the device were improved mainly at stall conditions, and flow separation was dumped even at high incidence. Similar controlling device was applied to HAWT blade in the numerical research conducted by Wang et al. [10]. It was concluded that for an appropriate rod settings, the steady off-surface VG was able to increase the blade torque up to $27.3 \%$ despite being at stall regime by effectively suppressing the separated flow.

The experimental studies mentioned above showed the benefits of small cylinder as passive control device in eliminating the separation bubble on bluff bodies and enhancement of aerodynamic performances of stalled wind turbines blades at high incidence. Consequently, a much smaller rod diameter was adopted in the current study to control the flow around NACA0012 wing at low Reynolds number of $4.45 \times 10^{5}$, where laminar and transitional boundary layer phenomena were clearly manifesting. The physical mechanisms of the flow control approach were examined by performing numerical investigation based on 3D RANS simulations. In addition, transitional effects where included in calculations to emphasize their importance in such flow conditions by considering transition sensitive closure turbulence model, $\gamma-\mathrm{Re}_{\theta, \mathrm{t}}$, rather than classical fully turbulence formulations.

\section{Passive device and simulation details}

\subsection{Wing model}

As previously indicated, the current work investigates the flow control around NACA0012 aerofoil wing using an off-surface micro cylinder device. The availability of experimental data to validate the computational model adopted here is one of the primary benefit of working with such standard symmetrical aerofoil. Furthermore, the physical mechanism of the interaction between the bodies is well predicted by simulating transitional effects through the use of transition sensitive RANS closure model, which is in the line with the current study's goal. Figure 1.b shows the overall geometric dimensions of the system as the chord, $\mathrm{c}=150 \mathrm{~mm}$, the span, $\mathrm{s}=3 \times \mathrm{c}$ and the rod diameter is, $\mathrm{d} / \mathrm{c}=1.34 \%$.

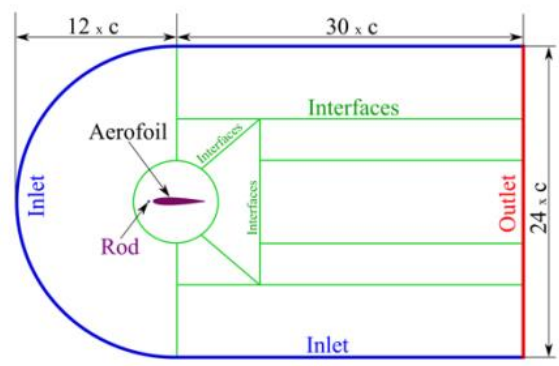

(a)

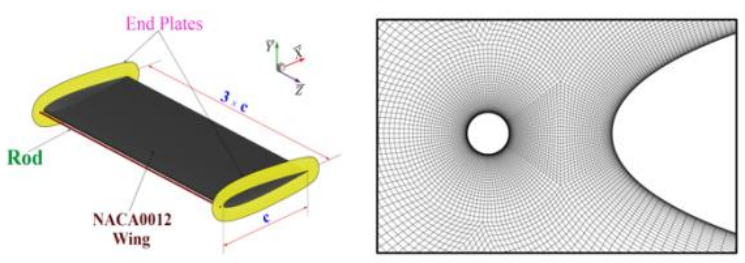

(b)

(c)

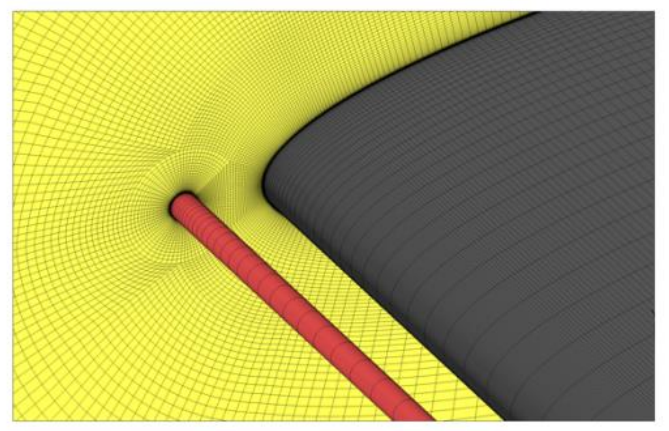

(d)

Fig. 1. (a) Computational domain schematic, (b) Rod-wing CAD model, (c) Meshing topology between rod and wing, (d) 3D structured mesh close to the rod. 


\subsection{Boundary conditions}

The computational domain is $42 \times \mathrm{c}$ long, $24 \times \mathrm{c}$ in height and $3 \times \mathrm{c}$ wide, as illustrated in Fig.1.a. In order to increase the mesh quality, reduce its size far from the wing and allow to change the angle of attack easily, a resolution box (disc cylinder) is built around the wing with dimension of $4 \times c$ along with mesh interfaces to optimize the element distribution, the blockage level for such configuration is around $2.7 \%$. Thus, with low blockage level and sufficient domain length it is fine to held the outlet boundary as constant pressure as it is not influenced by the wing. The Direct let boundary condition type of constant velocity is set at the inlet boundary to give a Reynolds number based on the chord length of $4.45 \times 10^{5}$. The two lateral boundaries are defined as a symmetry sides, and two endplates where incorporated to the wing sides as no-slip walls, thus any possible vortical tip flow is avoided when computing the flow over the model. For both rod and wing surfaces, the no slip boundary walls are specified. Under the flow conditions stated earlier, the Reynolds number based on the rod diameter is $\mathrm{Re}_{\mathrm{d}}=5931$. Thus, the formation length of the vortices behind this tiny cylinder is roughly equals to $2 \times \mathrm{d}$ [12]. Consequently, as the off-surface vortex generator is going to be placed in the vicinity of the leading edge, it is important to take into account this factor. Thus, the geometric arrangement of the control device is set at $3 \times \mathrm{d}$ upstream the wing to allow vortices generated in the wake of the rod to interact with the boundary layer of the suction surface.

\subsection{Turbulence model}

Steady RANS simulations have been carried out to understand the effect of the off-surface control device on the wing performances working at moderate Reynolds number where laminar effects are clearly manifesting. Given this overall aim, it is important to show the benefits of using transition sensitive closure model, $\gamma-\mathrm{Re}_{\theta t}$, in modelling flows with laminar induced separation. Menter et al. [13] have modified the formulation of $\mathrm{k}-\omega \mathrm{SST}$ turbulence model to determine the natural flow onset transition. The standard transport equations are coupled with another two equations for intermittency, $\gamma$ (Eq.1) and momentum thickness for Reynolds number transition, $\operatorname{Re}_{\theta, t}$ (Eq.2) which are combinated with experimental correlations.

$$
\begin{gathered}
\frac{\partial(\rho \gamma)}{\partial t}+\nabla \cdot(\rho U \gamma)=\nabla \cdot\left[\left(\mu+\frac{\mu_{t}}{\sigma_{\gamma}}\right) \nabla \gamma\right]+P_{\gamma} \\
-D_{\gamma} \\
\begin{array}{c}
\frac{\partial\left(\rho \widetilde{R e}_{\theta, t}\right)}{\partial t}+\nabla \cdot\left(\rho U \widetilde{R e}_{\theta, t}\right)= \\
+\cdot\left[\left(\mu+\frac{\mu_{t}}{\sigma_{\theta, t}}\right) \nabla \widetilde{R e}_{\theta, t}\right] \\
+P_{\theta, t}
\end{array}
\end{gathered}
$$

\subsection{Computational grid}

The computational domain is split into multiple blocks in order to create a high-quality structured mesh the system. This topology allowed to have finer mesh in sensitive regions where different size eddies are generated and coarse grid in far field. To satisfy these previous requirements. interface boundary conditions between various blocks are used during blocking construction. Furthermore, an O-grid topology is used around both the wing and the cylindrical rod to minimize overall skewness and provide improved cells orthogonality for the near wall grid elements when density is raised for the subsequent mesh dependency study (Fig.1.c and d). In addition, the mesh refinement at both rod airfoil gap and airfoil suction side is given special attention in order to capture precisely flow physical mechanisms of this passive control. Thus, it was concluded during this grid dependence investigation, 450 nodes around the wing and 180 nodes in the circumferential of the rod gave a fine enough mesh that eliminates the mesh dependency. Another important parameter during the mesh generation is to keep attention to the vulnerability of the transitional turbulence model to $\mathrm{y}^{+}$values. In order to produce realistic outcomes, a value of $\mathrm{y}^{+}$close to unity is required for the turbulence model to capture accurately the transition behavior of the flow. Consequently, based on flow velocity and typical length of the wing a target value of $\mathrm{y}^{+}=0.7$ was set and after calculations its maximum value was checked to be less than 1 in the post processing.

\subsection{Numerical model}

Steady RANS, incompressible, segregated solver was adopted in ANSYS-Fluent along with the transitional four equations $\gamma-\operatorname{Re}_{\theta t}$ closure model. The pressurevelocity field coupling was achieved using SIMPLE algorithm. Green-Gauss-Node based scheme was set to compute the gradients between cells. The third order MUSCL scheme was employed for spatial discretization of momentum equation, standard scheme was set for pressure and for all remaining equations a second order Upwind scheme was adopted. The inlet turbulence intensity was selected to provide freestream turbulence level of $0.3 \%$ close to the studied system as recommended by ANSYS-fluent guide [14], as the freestream turbulence intensity decreases throughout the length of the computational domain due to dissipation terms in numerical transport equations. All different cases were initialized with constant inlet velocity and computed up to 50000 iterations where scaled residuals for all equations were decreased to the order of $10^{-6}$, by which time the monitored aerodynamic coefficients had become steady.

\subsection{CFD validation}

For a better prediction of the aerodynamic coefficients mesh study was carried out to validate the numerical model by comparing the results to the experimental work of Gregory and O'Reilly (1970) [11] who carried 
out experiments on NACA0012 airfoil wing for different angles of attack and Reynolds numbers. The comparison is based on the pressure coefficients on the suction surface of the wing at $10^{\circ}$ incidence. The Reynolds number for which the investigation is done is $2.88 \times 10^{6}$. Figure 2 shows the concordance of the current numerical results with the experimental data presented by authors [11].

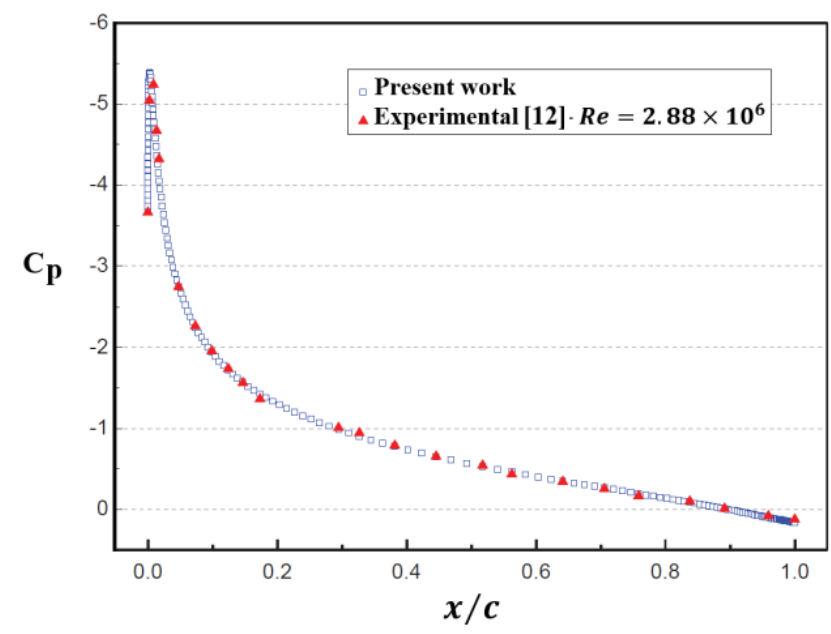

Fig. 2. Pressure coefficient at suction side of the wing.

\section{Results and discussion}

Figure.3.a, shows the average velocity over the wing span midline section for the baseline configuration. For $\alpha \leq 5^{\circ}$, the mean velocity is positive and takes the same direction as the incident flow, from the leading edge to the trailing edge. For $\alpha>5^{\circ}$, a return flow zone begins to appear at the trailing edge going towards the leading edge with as $\alpha$ increased. This area is indicated by negative average velocity. This reveals that the turbulent separation of the boundary layer occurs gradually as it moves towards the leading edge. For $\alpha=12^{\circ}$ and $\alpha=$ $13^{\circ}$, the negative velocity zone suddenly expands and spreads almost over the entire suction side, especially for $\alpha=13^{\circ}$. This phenomenon indicates a severe turbulent stall that is occurring. The boundary layer is competently peeled off from the wall. The thickness of the wake becomes very large compared to low angles of attack. For $\alpha>13^{\circ}$, the return flow amplifies and the thickness of the wake widens further with increasing AOAs. The negative mean velocity is spread over the entire wing upper surface. Figure.3.b, shows the average speed taken on the center line of the wingspan at suction side for the controlled case, in the presence of the off surface vortex generator in form of cylinder of dimension, $\mathrm{d} / \mathrm{c}=1.34 \%$ located at $3 \times \mathrm{d}$ from the leading edge to its centerline. For $\alpha \leq 8^{\circ}$, it can be seen that the mean velocity is positive and takes the direction of the incident flow (from the leading edge to the trailing edge). The thickness of the wake developed behind the wing remained thin with the increase of AOAs. From $\alpha$ $=10^{\circ}$ up to $\alpha=14^{\circ}$, instability is triggered at the trailing edge, indicated by the appearance of negative average velocity. This instability suddenly amplifies between $\alpha$ $=14^{\circ}$ and $\alpha=15^{\circ}$. For $\alpha=15^{\circ}$, the negative mean velocity field occupies the entire length of the wing. Hence, a sudden change in the dimensions of the wake is observed. The thickness of the wake widens significantly, indicating the turbulent release of the boundary layer. This phenomenon reveals the existence of a vortex burst caused by the amplification of instability triggered at the trailing edge previously detected for low incidences. For $\alpha>15^{\circ}$, the return flow develops a recirculation which amplifies to thicken the wake which widens further by increasing the angle of attack. To sum up, compared to the uncontrolled case, where the sudden widening of the wake is detected for $\alpha=13^{\circ}$, the phenomenon is observed for $\alpha=15^{\circ}$. Therefore, the turbulent stall of boundary layer is pushed back to higher angles of incidence. Consequently, this control case pushes the turbulent stall angle to higher angles as can be seen for $\alpha=15^{\circ}$. For the nominal case (Fig.3.a), it was observed that for very low angles of attack, the friction lines are uniform and straight flowing from the leading edge towards the trailing edge (same direction as that of the incident flow). On both ends of the trailing edge, a little instability begins to set in.

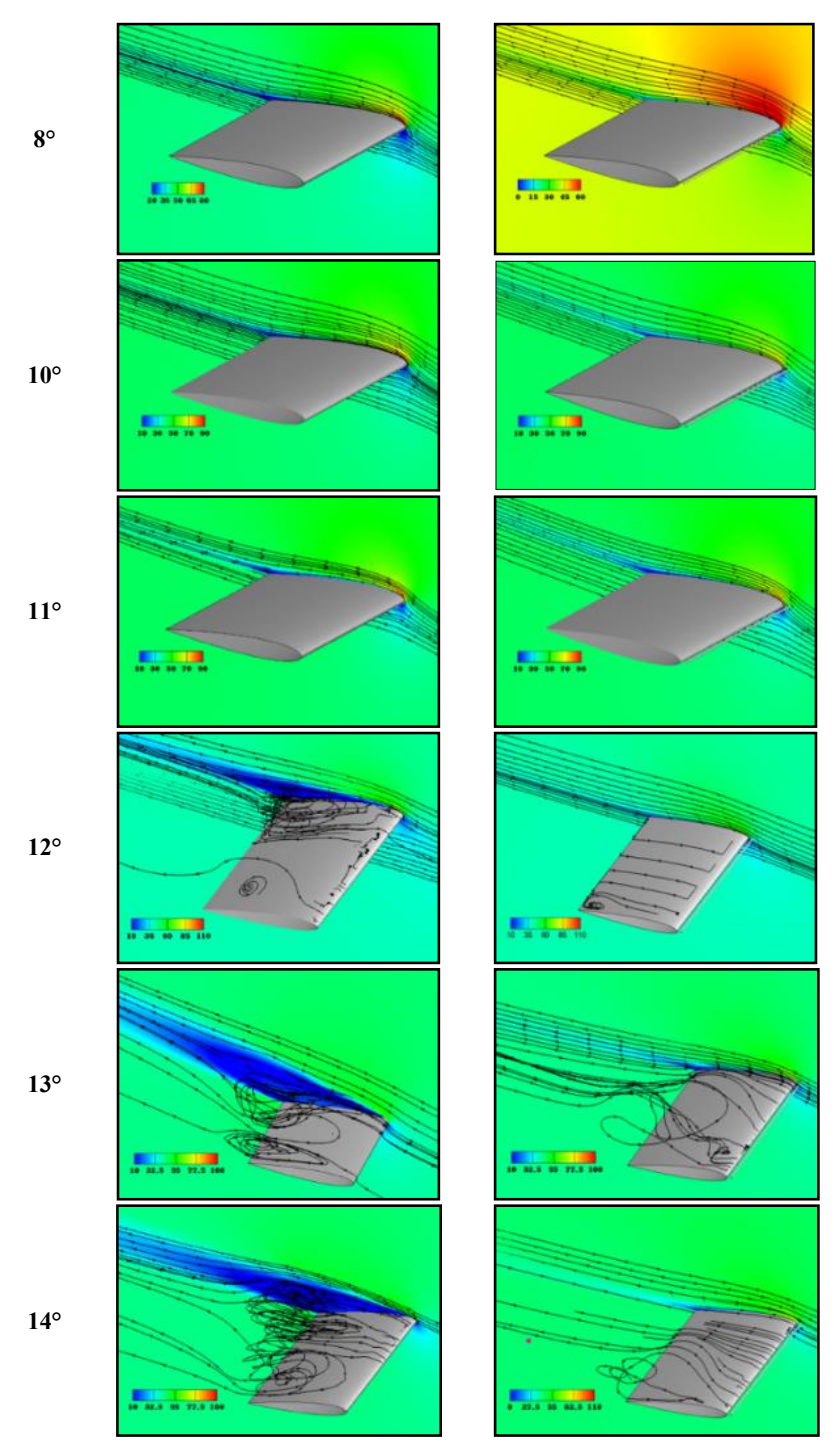


$15^{\circ}$

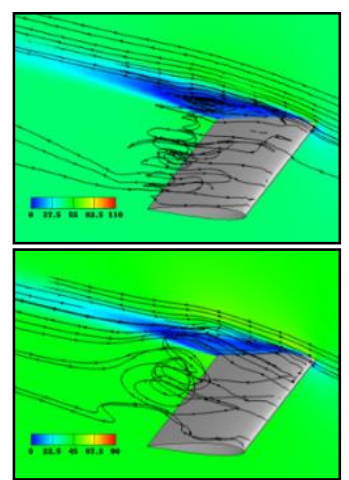

(a)

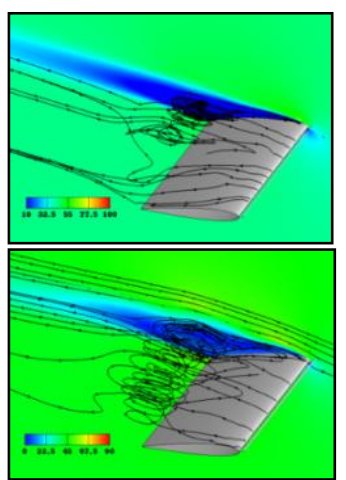

(b)
Fig. 3. Mean velocity magnitude-streaklines, (a) baseline case, (b) Controlled case (flow moving right to left).

As the incidence increased $\left[0^{\circ}-11^{\circ}\right]$, this instability gradually amplifies as it moves from the trailing edge to the leading edge. Thus, these friction lines lose their straightness and uniformity. This instability indicates the presence of a partial stall of the boundary layer. Furthermore, the phenomenon gradually amplifies with the increase of $\alpha$ to form two large contra-rotating vortices (Fig.3.a. $\left(\alpha=11^{\circ}\right)$ ). Indeed, the presence of these two vortices causes the birth of a return flow on the two ends of the wing suction side, while the flow field, between these two vortices, maintains the same direction than that of incident flow. This reveals that the energy of the incident flow is greater compared to that of the return flow, because, the incident flow is dominant and imposes its presence in the middle zone, compared to the return flow in which the boundary layer is not completely detached. Thereby, the boundary layer stall only occurred in areas where the two counterrotating vortices are present. For $\alpha=12^{\circ}$, the direction of the friction lines between the two primary vortices is reversed. The size of the two counter-rotating vortices has been reduced. The energy of the return flow becomes significant compared to that of the incident flow. Therefore, the interaction of the two opposite direction flows causes the appearance of secondary instabilities. The coiling of the friction lines shows that the birth of the counter-rotating primary vortices is due to the coiling of the friction lines of the incident flow. The existence of friction lines having the same direction as that of the incident flow shows that the boundary layer is not completely detached. For $\alpha=13^{\circ}$, the return flow dominates over the entire upper surface as the counter-rotating vortices exist and wind up depending on the return flow. Consequently, the direction of winding of these two primary vortices is reversed compared to the previous cases. For $\alpha=14^{\circ}$ and $\alpha=15^{\circ}$, it canbe clearly seen that the return flow becomes completely dominant. The friction lines run from the trailing edge to the leading edge. The two counterrotating vortices move away from each other to develop on both ends of the upper surface. Hence, their intensities weaken compared to that observed for the previous cases. For $\alpha>15^{\circ}$, the friction lines show that the return flow is established over the entire upper surface. The boundary layer is completely detached. The two counter-rotating vortices have disappeared and the flow within the boundary layer is moving from the trailing edge to the leading edge with the presence of weak disturbances on the upper surface. In addition, the interaction between the two flows, incident and return, is limited only to the leading edge.

It can be seen also from Figure.3.b, that streak lines on the wing suction side for the controlled case using the micro-cylindrical rod of diameter $\mathrm{d} / \mathrm{c}=1.34 \%$, where several angles of attack are considered. For $\alpha<5^{\circ}$, the streak lines on the wall take the same direction as that of the incident flow with uniform and perfectly linear behaviour. For $5^{\circ} \leq \alpha \leq 13^{\circ}$, two primary instabilities begin on both ends of the leakage edge. These instabilities gradually increase with the increase in the angle of attack. This phenomenon reveals a partial stall of the boundary layer that starts from the trailing edge by progressively propagating to the leading edge, as $\alpha$ increases.

Accordingly, the amplification of these primary instabilities leads to a sudden change in the shape of a swirling burst, marked by the appearance of the two contrarotative vortices, which occupy most of the upper surface. This phenomenon is well established for $\alpha=$ $14^{\circ}$. This means that the return flow is not powerful enough to oppose the incident flow. For $\alpha=15^{\circ}$ and $\alpha=$ $16^{\circ}$, the return flow dominates the middle portion of the upper surface indicating a total breakout of the boundary layer. The two vortices move away from each other, expanding on the tips of the wing, and their dimensions are reduced compared to those observed for $\alpha=14^{\circ}$. The formation of the primary vortices is caused by the coiling of the streak lines of the incident flow. For $\alpha>$ $16^{\circ}$, the return flow is established over the entire surface of the wing. These lines run from the trailing edge to the leading edge without rolling up, except for a few small deformations near the leading edge which are caused by interaction with the incident flow. Indeed, the two contrarotating primary vortices have completely disappeared. Thus, this indicates that the boundary layer is completely detached from the wall. The primary contra-rotating vortices are observed for $\alpha=14^{\circ}$. This means that, for a dimension of $\mathrm{d} / \mathrm{c}=1.34 \%$, the onset of this phenomenon is delayed by, $\Delta \alpha=3^{\circ}$, compared to the baseline case.

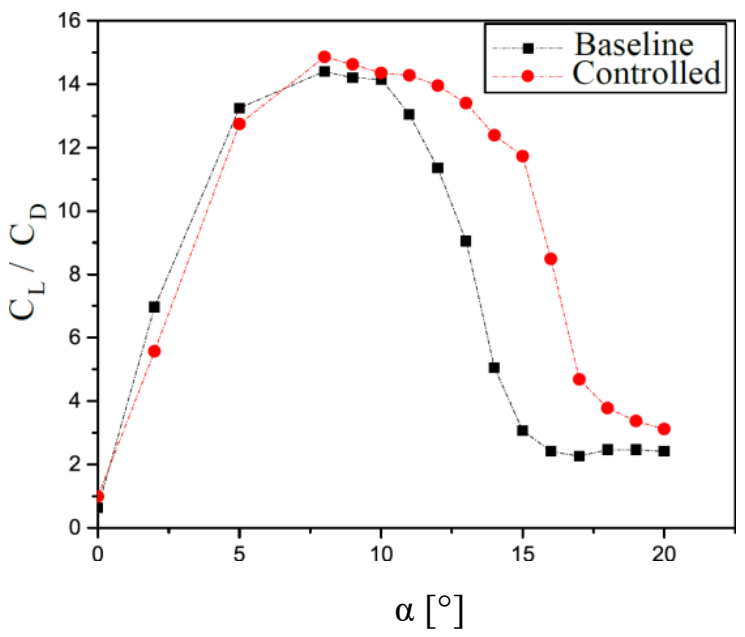

Fig. 4. Lift to Drag ratio as function of AOAs. 
Figure.4, depicts the lift to drag ratio $\left(\mathrm{C}_{\mathrm{L}} / \mathrm{C}_{\mathrm{D}}\right)$ as the incidence is varied for both baseline and controlled cases. It can be seen that both curves are initiated by rapid raise in lift to drag ratio for low angles of attack at pre-stall regime until reaching a maximum value regardless whether the rod is introduced to the flow or not. However, a slight loss in lift to drag ratio is observed when the angle of attack, $\alpha$ lies between $0^{\circ}$ to $8^{\circ}$ that is mainly due to the off surface rod wake that generates an additional drag compared to the baseline wing. After that, the lift to drag ratio is then gradually reduced until it reaches the post-stall regime, at which a significant loss in $C_{L} / C_{D}$ ratio is manifesting due to the stall phenomenon. At post stall regime, the increase in angles of attack has no effect on lift to drag ratio as both curves tended to be constant. Moreover, it is important to point out the ineffectiveness of the considered control device for angles of incidence less than $10^{\circ}$, where no substantial improvement in $\mathrm{C}_{\mathrm{L}} / \mathrm{C}_{\mathrm{D}}$ ratio is recorded. Whereas, as AOA lies between $11^{\circ}$ to $16^{\circ}$ the gain is noteworthy and varies from $22.68 \%$ to $134.17 \%$ when micro off-surface cylinder is placed in the vicinity of the leading edge compared to the baseline configuration. According to the last result, it is imperative to study the response of the airfoil while varying the position, dimension and shape of the vortex generator in order to improve the aerodynamic performances of the wing in its varieties of use.

\section{Conclusions}

The objective of this study is to numerically investigate the strategy of the off-surface passive flow control around a wing with NACA0012 airfoil at moderate Reynolds number, $\mathrm{Re}=4.45 \times 10^{5}$. A micro-cylinder vortex generator was placed upstream the leading edge at an optimum position of $3 \times \mathrm{d}$ seeking for high performance and a diameter of, $\mathrm{d} / \mathrm{c}=1.34 \%$. It was mainly noticed that this control device acts similarly to a pumping system energizing the boundary layer on the airfoil counter-balancing the adverse pressure gradients. Hence, It showed a capability of maintaining attached flow for angle of attack of $15^{\circ}$ up to $33 \%$ of its chord length which was confirmed from flow structure analysis. Moreover, at post-stall regime the rod seemed to effectively suppress or diminish and in most favorable case delay flow separation to higher incidences, which is advantageous to either lift enhancement or drag reduction, resulting in significant improve in lift to drag ratio up to $135 \%$ at post stall flow conditions.

\section{References}

1. Gad-el-Hak, M. (2007), Flow control: passive, active, and reactive flow management, Cambridge university press.

2. Strykowski, P. J., and Sreenivasan, K. R. (1990), On the formation and suppression of vortex'shedding' at low Reynolds numbers, Journal of Fluid Mechanics, 218(1), pp. 71-107.

3. Sakamoto, H., Tan, K., and Haniu, H. (1991), An optimum suppression of fluid forces by controlling a shear layer separated from a square prism, Journal of Fluids Engineering, 113 (2), pp. 183-189.

4. Sakamoto, H., and Haniu, H. (1994), Optimum suppression of fluid forces acting on a circular cylinder, Journal of Fluids Engineering, 116, pp. 221-227.

5. Igarashi, T. (1997), Drag reduction of a square prism by flow control using a small rod, Journal of Wind Engineering and Industrial Aerodynamics, 69, pp. 141-153.

6. Tsutsui, T., and Igarashi, T. (2002), Drag reduction of a circular cylinder in an air-stream, Journal of Wind Engineering and Industrial Aerodynamics, 90(4-5), pp. 527-541.

7. Michelis, T., and Kotsonis, M. (2015), Interaction of an off-surface cylinder with separated flow from a bluff body leading edge. Experimental Thermal and Fluid Science, 63, pp. 91-105.

8. Hayder, K., and Rashid, N. (2013), Numerical and experimental investigation of co-shedding vortex generated by two adjacent circular cylinders and its effect on air flow behavior around Naca 2412 airfoil, Industrial Engineering Letters, 3 (6), pp. 34-45.

9. Chen, J., Sun, X. J., and Huang, D. G. (2015), A new type of vertical-axis wind turbine equipped with the blades having micro-cylinders installed in front of their leading-edges, Journal Engineering Thermophysics, 1, pp. 75-78.

10. Wang, Y., Li, G., Shen, S., Huang, D., and Zheng, Z. (2018), Investigation on aerodynamic performance of horizontal axis wind turbine by setting microcylinder in front of the blade leading edge, Energy, 143, pp. 1107-1124.

11. Gregory, N., and O'reilly, C. L. (1970), Low-speed aerodynamic characteristics of NACA 0012 aerofoil section, including the effects of upper-surface roughness simulating hoar frost, Aeronautical Research Council R \& M, No. 3726.

12. Norberg, C. (1998), LDV-measurements in the near wake of a circular cylinder, Proceedings of the conference on bluff body wakes and vortex induced vibration, Washington DC, Edited by P.W. Bearman and C.H.K. Williamson (Cornell University, Ithaca, NY), pp.1-12.

13. Menter, F. R., Langtry, R., and Völker, S. (2006), Transition modelling for general purpose CFD codes, Flow, turbulence and combustion, 77 (1-4), pp. 277-303.

14. ANSYS Fluent Tutorial Guide, ANSYS, Inc. January 2017 Southpointe 2600 ANSYS Drive Canonsburg, PA 15317, ansysinfo@ansys.com, http:// www.ansys.com, registered ISO 9001: 2008 companies. 